\title{
White Matter Microstructure and the Variable Adult Outcome of Childhood Attention Deficit Hyperactivity Disorder
}

\author{
Philip Shaw*, 1,2,4, Gustavo Sudre',4, Amy Wharton', Daniel Weingart', Wendy Sharp ${ }^{2}$ and Joelle Sarlls ${ }^{3}$ \\ 'Section on Neurobehavioral Clinical Research, Social and Behavioral Research Branch, National Human Genome Research Institute, Bethesda, \\ MD, USA; ${ }^{2}$ Intramural Program of the National Institute of Mental Health, Bethesda, MD, USA; ${ }^{3}$ NIH MRI Research Facility/National Institute of \\ Neurological Diseases and Stroke/National Institutes of Health, Bethesda, MD, USA
}

\begin{abstract}
Changes in cerebral cortical anatomy have been tied to the clinical course of attention deficit hyperactivity disorder (ADHD). We now ask if alterations in white matter tract microstructure are likewise linked with the adult outcome of childhood ADHD. Seventy-five young adults, 32 with ADHD persisting from childhood and 43 with symptom remission were contrasted against 74 never-affected comparison subjects. Using diffusion tensor imaging, we defined fractional anisotropy, a metric related to white matter microstructure, along with measures of diffusion perpendicular (radial) and parallel (axial) to the axon. Analyses were adjusted for head motion, age and sex, and controlled for multiple comparisons and medication history. Tract-based analyses showed that greater adult inattention, but not hyperactivity-impulsivity, was associated with significantly lower fractional anisotropy in the left uncinate (standardized $\beta=-0.37$, $t=3.28, p=0.002$ ) and inferior fronto-occipital fasciculi (standardized $\beta=-0.37, t=3.29, p=0.002$ ). The ADHD group with symptoms persisting into adulthood had significantly lower fractional anisotropy than the never-affected controls in these tracts, differences associated with medium to large effect sizes. By contrast, the ADHD group that remitted by adulthood did not differ significantly from controls. The anomalies were found in tracts that connect components of neural systems pertinent to ADHD, such as attention control (inferior fronto-occipital fasciculus) and emotion regulation and the processing of reward (the uncinate fasciculus). Change in radial rather than axial diffusivity was the primary driver of this effect, suggesting pathophysiological processes including altered myelination as future targets for pharmacological and behavioral interventions.

Neuropsychopharmacology (20I5) 40, 746-754; doi:I 0.I038/npp.20I4.24I; published online I5 October 20I4
\end{abstract}

\section{INTRODUCTION}

ADHD is far from being a problem confined to childhood, but rather it persists into adulthood in $20-40 \%$ of individuals (Faraone et al, 2006). Problems with attention tend to be more prominent than hyperactivity-impulsivity in adults and are a major cause of impairment for the individuals in the workplace and in interpersonal relationships (Birnbaum et al, 2005; Faraone et al, 2006; Pingault, 2011). Understanding the neural basis of the variable outcome is not only a public health priority but could also help stimulate novel treatment approaches to promote remission from ADHD.

ADHD is increasingly conceptualized as the result of disruption to multiple neural systems rather than reflecting

*Correspondence: Dr P Shaw, Section on Neurobehavioral Clinical Research, Social and Behavioral Research Branch, National Human Genome Research Institute, 9000 Rockville, Building 31, BI B37, Bethesda, MD 20892, USA, Tel: + I 30| 45। 40I0, Fax: + I 30| 480 3I08,

E-mail: shawp@mail.nih.gov

${ }^{4}$ These authors contributed equally to this work

Received 2 July 2014; revised II August 2014; accepted 16 August 20।4; accepted article preview online 22 September 2014 dysfunction within one neuropsychological domain (Casey and Durston, 2006; Castellanos and Proal, 2012; Durston et al, 2011; Nigg and Casey, 2005; Sonuga-Barke, 2005). Thus it is likely that the variable clinical course of the disorder will reflect the development of multiple neural systems. In support of this concept, we recently found that severity of adult inattentive symptoms of ADHD was linked with differing developmental trajectories within the cortical components of multiple neural networks supporting attention, cognitive control, and the default mode network (Shaw et al, 2013). A logical next step is to examine the properties of the white matter tracts that constitute the structural connections within these neural systems. This can be attained using diffusion tensor imaging (DTI), which probes tissue microstructure through the diffusion of water within the brain (Basser et al, 1994). The technique provides measures of white matter microstructure such as fractional anisotropy (FA), and more focused measures, specifically diffusion parallel (axial diffusivity) and perpendicular (radial diffusivity) to axons (Basser and Pierpaoli, 1996). FA is determined by a complex amalgam of the physical determinants of white matter microstructure, including axonal packing, myelination, and the coherence of fiber 
direction (Basser and Pierpaoli, 1996). Although a direct mapping from FA to a specific tissue characteristic is thus not possible, the metric does capture important aspects of white matter architecture that allow us to delineate the physical basis for structural connectivity.

White matter tract morphology is also of interest to those seeking to define the neural processes related to the variable clinical outcome of neurodevelopmental disorders such as $\mathrm{ADHD}$. It is possible that the degree of disruption to white matter microstructure in adults with a history of childhood ADHD will reflect the severity of current adult symptoms. In testing this model, an approach that treats symptoms as a continuous variable is preferred as this maximizes power to detect symptom-related changes. This approach also allows a separate examination of the symptom dimensions that is necessary given their different natural histories and our finding that cortical development is linked with adult inattention but not hyperactivity-impulsivity. A second possibility is that a history of childhood ADHD leaves a 'mark' on white matter microstructure that persists regardless of the course of ADHD in adulthood. The only study to examine adults with a variable outcome from their childhood ADHD found such remnants of childhood status when data were analyzed on the basis of childhood rather than adult diagnostic status (Cortese et al, 2013). An even more stringent approach is to define trait markers in white matter as the anomalies that are found even among adults who have remitted from ADHD. In these adults the contribution of 'state'-related anomalies reflecting current symptom status is negated, helping dissect out the contribution of an enduring 'trait'. Here we employ both the approaches.

Progress has been made in charting the neurocognitive basis of the variable clinical course of ADHD. Change in effortful, executive function has been found to track with symptom severity, whereas lower-level processes may reflect the onset of $\mathrm{ADHD}$ regardless of its course (Rajendran et al, 2013). This link between cognition and clinical course may be more pronounced in early rather than late childhood (Coghill et al, 2014), and this developmental heterogeneity may explain the limited power of cognitive measures in predicting outcome (van Lieshout et al, 2013). Neuroanatomic imaging studies generally find that cerebral, cerebellar, and hippocampal anatomies are more atypical in adults with persisting symptoms and tend to normalize in those who remit (Halperin and Schulz, 2006; Mackie et al, 2007; Plessen et al, 2006; Shaw et al, 2013). This normalization may be only partial and thus some anomalies that reflect a childhood history of ADHD have been reported in adults (Cortese et al, 2013; Proal et al, 2011). The functional imaging literature similarly finds prominent state-related anomalies sometimes against a background of trait deficits. Thus, whereas decreased thalamo-cortical activation during response preparation in adults reflected their childhood history of ADHD, the level of coordinated activity within this network was linked with the severity of their current, adult symptoms (Clerkin et al, 2013). Similarly, anomalous connectivity within the default mode network in adults reflected both the severity of current ADHD symptoms and a childhood history of ADHD, depending on the region chosen as the center of the network (Mattfeld et al, 2014).
Here, we aim to extend this work by defining white matter microstructure in a group of young adults who have a variable outcome from their childhood ADHD. First, we asked whether anomalies in white matter microstructure would be 'state related', associated with the severity of adult symptoms of inattention rather than hyperactivityimpulsivity, as we found for cortical development (Shaw et al, 2013). Second, we tested for possible 'trait' markers that could reflect the history of childhood ADHD in this adult cohort. Finally, we explored whether change in radial or axial diffusivity was more prominent as these measures point to partly distinct pathophysiological mechanisms.

\section{MATERIALS AND METHODS}

Participants were drawn from a longitudinal ongoing study into brain development at the intramural programs of the National Institutes of Health. This imaging study examined the adult status of 75 individuals with a childhood history of ADHD. Sixty-four entered the study as children and a childhood diagnosis of DSM-IVR ADHD had been determined using the Parent Diagnostic Interview for Children and Adolescents (Reich, 2000). For the 11 participants who entered the study as young adults, collateral information to confirm a history of childhood ADHD was obtained from parents or health-care providers. For all individuals, the assessment of adult ADHD symptoms was obtained through clinical interviews (either PS or WS) using the clinicianadministered ADHD Rating Scale, version IV, providing examples and prompts appropriate for late adolescent and young adult groups (DuPaul et al, 1998). The interviewer rated each of the nine possible symptoms of inattention and nine symptoms of hyperactivity/impulsivity. In line with DSM-5, attention deficit/hyperactivity disorder, combined presentation, was diagnosed when an individual has both five or more symptoms of inattention and five or more symptoms of hyperactivity/impulsivity. The inattentive or hyperactive/impulsive presentations are diagnosed when symptoms are confined to these domains. Presence of other psychiatric diagnoses was established through the Structured Clinical Interview for DSM Axis I Disorders. Medication histories were obtained from participants.

Contrasts were made against 74 subjects who never had $\mathrm{ADHD}$, referred to as the comparison group, who were drawn from a study of typical brain development. The $\mathrm{ADHD}$ and comparison groups were matched on sex, age of assessments, and intelligence. All comparison subjects were free of Axis I DSM-IV mental disorders. Intelligence quotient was estimated using age-appropriate versions of the Wechsler intelligence scales. General exclusion criteria were a full-scale IQ of $<80$, evidence of neurological disorders known to affect brain structure, current substance dependence, or psychotic disorders. Institutional review boards of the National Institutes of Health approved the research protocol, and written informed consent was obtained from participants.

\section{Neuroanatomic Methods}

All data were collected on a 3-T HDx MRI system (GE Healthcare, Milwaukee, WI) with gradients capable of 
$40 \mathrm{mT} / \mathrm{m}$ and using an eight-channel phase array coil. DTI data were acquired with a single-shot dual-spin-echo echo-planar imaging sequence, with the following parameters: $\mathrm{TE}=85 \mathrm{~ms}, \mathrm{TR}=18.5 \mathrm{~s}, \mathrm{FOV}=240 \mathrm{~mm}, 96 \times 96$ matrix, slice thickness $=2.5 \mathrm{~mm}$, gap $=0 \mathrm{~mm}$, and acceleration factor $=2$. A custom set of diffusion directions and weightings were acquired for a total of 80 volumes: 10 volumes at $b$-value $=0 \mathrm{~s} / \mathrm{mm}^{2}, 10$ volumes with evenly distributed directions at $b$-value $=300 \mathrm{~s} / \mathrm{mm}^{2}$, and 60 volumes with evenly distributed directions at $b$-value $=$ $1100 \mathrm{~s} / \mathrm{mm}^{2}$.

The diffusion data were processed using the TORTOISE software package (Pierpaoli et al, 2010). Preprocessing steps included rigid body motion correction, eddy-current distortion correction, B0 distortion correction, and bicubic upsampling to $1.5 \mathrm{~mm}$ isotropic resolution. The diffusion tensor was computed by nonlinear least squares fitting (Basser et al, 1994). All data were visually inspected and individuals who had more than one volume showing artifacts were excluded. The degree of head motion was determined from the absolute value of the three translation parameters that were averaged over the registered volumes and combined using the Euclidean norm. A similar procedure defined a rotation index for each subject. Data sets were excluded if there was $>3 \mathrm{~mm}$ of combined translation or $>0.03$ radians of rotation. In addition, data sets with extreme radial or axial diffusivity values in white matter tracts were excluded. Finally, some typical subjects were removed to equate the groups in head motion, sex, and age. As a result, 149 of the initial 200 DTI data sets were retained.

DTI-TK software was used to register the diffusion tensors into a common adult template space (Zhang et al, 2007; Zhang et al, 2009). This spatial normalization software uses the directional information of the diffusion tensors to align the data of all subjects, incorporates sequential rigid, affine, and nonlinear registration steps, and has been ranked as the top-performing tool in its class (Wang et al, 2011). Tract-specific analyses were also performed using this software (Zhang et al, 2010). This defines 11 major white matter tracts through alignment to a template that captures the average shape and diffusion properties of tracts parcellated via modeling of sheet-like white matter fasciculi (Zhang et al, 2010). This software calculates the diffusion measures locally (FA, radial diffusivity, and axial diffusivity), which are then projected onto a medial model, or a mesh of the tract, the model being a sheetlike structure that represents the 3D tract. We estimate the diffusion metric at the point with the maximum FA value along the spokes that correspond to each vertex of the mesh (similar to Tract-Based Spatial Statistics (Smith et al, 2006)). The 11 tracts examined were the bilateral uncinate, inferior fronto-occipital, superior longitudinal, inferior longitudinal, corticospinal fasciculi, and the corpus callosum.

\section{Analyses}

We regressed inattention and hyperactive-impulsive symptoms in the ADHD group against FA for the 11 tracts, and a Bonferroni correction for multiple comparisons was applied $(0.05 / 11=0.0045)$. In adjusted analyses, age, sex, and head motion parameters were entered as covariates; thus the model was:

$$
\begin{aligned}
\text { Tract FA } \sim & \beta_{0}+\left[\beta_{1} \times \text { symptom score }\right]+\left[\beta_{2} \times\right. \text { sex } \\
& (\text { male }=\text { reference })]+\left[\beta_{3} \times \text { head motion }\right]+\left[\beta_{4} \times \text { age }\right]
\end{aligned}
$$

In a further regression, the ADHD participants were categorized into persistent and remitted groups, and the FA values for each group were contrasted against the reference, never-affected comparison group. In these regressions, the never-affected group was taken as the reference category. Bonferroni adjustment was made for multiple comparisons (22 planned contrasts for FA; $p<0.0023$ ). Group differences were expressed as effect sizes (where $d \sim 0.2$ is a small, $d \sim 0.5$ is a medium, and $d \sim 0.8$ is a large effect size).

Finally, to facilitate comparison with previous studies (Proal et al, 2011), we reanalyzed the data on the basis of childhood diagnoses, comparing the group with a history of childhood ADHD against the never-affected comparison group.

\section{RESULTS}

\section{Clinical}

The comparison and clinical groups did not differ significantly in age, estimated intelligence, or sex (Table 1). Rates of daily psychostimulant were significantly higher and there was a trend to higher rates of comorbidity in the persistent compared with the remitted ADHD group.

\section{Tract-Based Analyses}

In dimensional analyses, there was a significant association between inattentive symptoms and decreased FA in the inferior fronto-occipital longitudinal fasciculi bilaterally and the left corticospinal and uncinate fasciculi-see Table 2 and Figure 1. These links survived adjustment for age, sex, and head motion within the left inferior frontooccipital and left uncinate fasciculi, with trends for the left corticospinal and right inferior fronto-occipital tracts. There were no significant associations between hyperactiveimpulsive symptoms and FA that survived correction for multiple comparisons. However, a nominally significant association emerged for the right superior longitudinal fasciculus (Supplementary Table 1).

Changes in radial diffusivity were the primary contributor to this link between white matter microstructure and inattentive symptoms-Table 2 . Thus, there were significant links between inattentive symptoms and radial diffusivity in both adjusted and unadjusted analyses for the bilateral inferior fronto-occipital, uncinate, and superior longitudinal fasciculi. A significant association between axial diffusivity and inattentive symptoms was found for the right inferior longitudinal fasciculus only.

In the planned categorical contrasts, the remitted ADHD group did not differ significantly from the never-affected comparison group in any tract-Table 3. However, the persistent ADHD group showed reduced FA relative to the never-affected comparison group in the bilateral uncinate fasciculi and the right inferior fronto-occipital fasciculus, which held following adjustment for age, head motion and 
Table I Demographic and Clinical Characteristics of the Participants

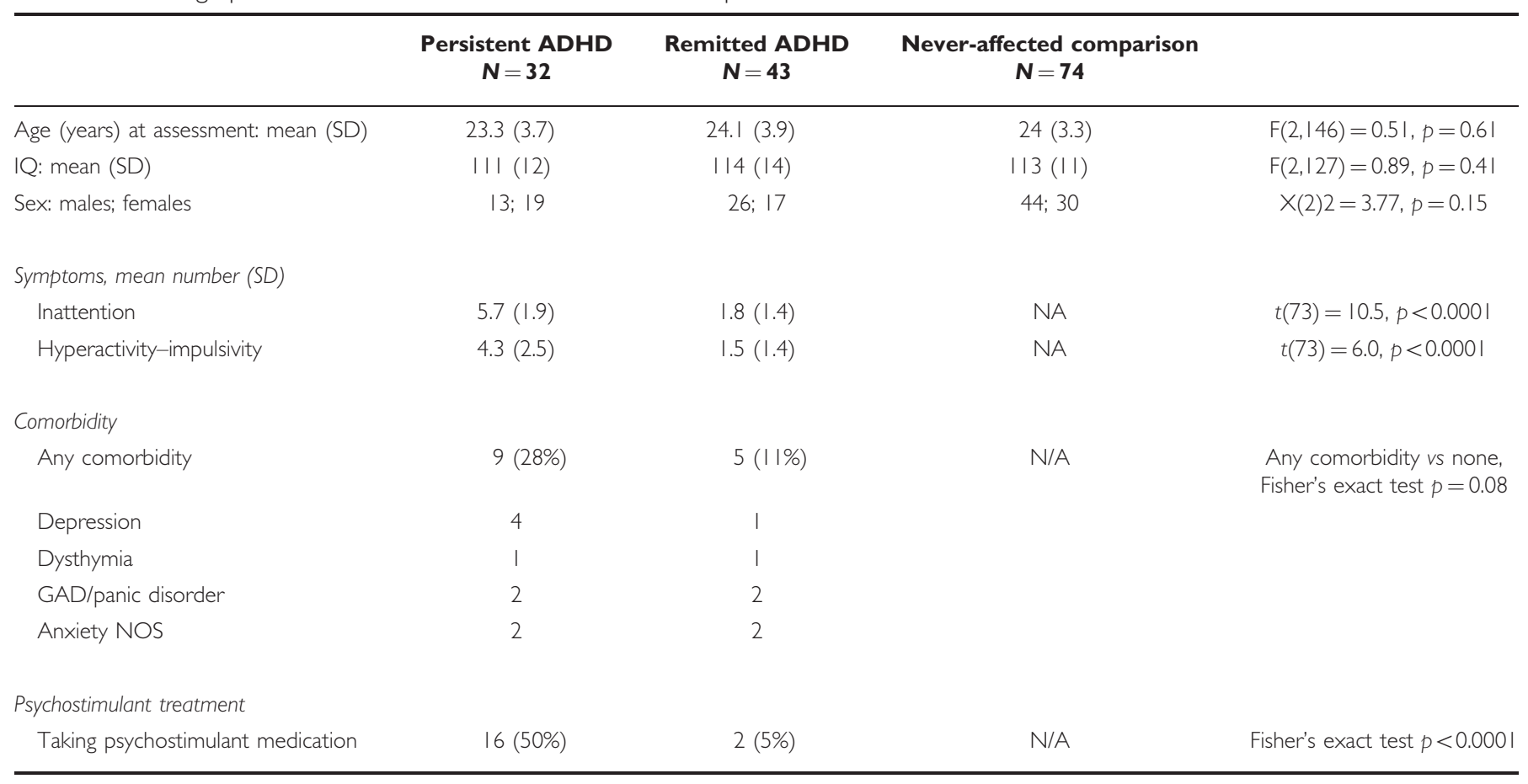

Abbreviations: GAD, Generalized anxiety disorder; NOS, not otherwise specified.

Missing data are indicated in the degrees of freedom.

Table 2 The Standardized $\beta$ Coefficients, Associated $t$ and $p$ Values for the Regression of Inattentive Symptoms in the Group with a History of Childhood ADHD against White Matter Metrics for the II Major Tracts

Tracts

Inattentive symptoms

\begin{tabular}{|c|c|c|c|c|c|c|c|c|c|c|c|c|}
\hline & \multicolumn{3}{|c|}{$\begin{array}{c}\text { Fractional anisotropy: } \\
\text { unadjusted }\end{array}$} & \multicolumn{3}{|c|}{$\begin{array}{c}\text { Fractional anisotropy: } \\
\text { adjusted }\end{array}$} & \multicolumn{3}{|c|}{$\begin{array}{l}\text { Radial diffusivity: } \\
\text { adjusted }\end{array}$} & \multicolumn{3}{|c|}{$\begin{array}{l}\text { Axial diffusivity: } \\
\text { adjusted }\end{array}$} \\
\hline & Std $\beta$ & $t$ & $P$ & Std $\beta$ & $t$ & $P$ & Std $\beta$ & $t$ & $\mathbf{P}$ & Std $\beta$ & $t$ & $P$ \\
\hline$L$ inferior fronto-occipital & -0.42 & 3.94 & $0.0002^{\mathrm{a}}$ & -0.37 & 3.29 & $0.002^{\mathrm{a}}$ & 0.36 & 4.53 & $0.0000 \mathrm{I}^{\mathrm{a}}$ & 0.14 & 1.57 & 0.12 \\
\hline $\mathrm{R}$ inferior fronto-occipital & -0.39 & 3.64 & $0.00 \mathrm{I}^{\mathrm{a}}$ & -0.32 & 2.71 & 0.009 & 0.35 & 4.26 & $0.0004^{\mathrm{a}}$ & 0.08 & 0.87 & 0.39 \\
\hline$L$ uncincate & -0.46 & 4.45 & $0.00003^{a}$ & -0.37 & 3.28 & $0.002^{a}$ & 0.35 & 4.43 & $0.00002^{a}$ & 0.12 & 1.42 & 0.16 \\
\hline L corticospinal & -0.35 & 3.17 & $0.002^{\mathrm{a}}$ & -0.33 & 2.84 & 0.006 & 0.10 & 1.21 & 0.23 & 0.06 & 0.69 & 0.49 \\
\hline R corticospinal & -0.31 & 2.77 & 0.007 & -0.23 & 1.98 & 0.05 & 0.05 & 0.62 & 0.53 & -0.03 & 0.38 & 0.7 \\
\hline L superior longitudinal & -0.30 & 2.65 & 0.01 & -0.26 & 2.1 & 0.04 & 0.30 & 3.55 & $0.00 \mathrm{I}^{\mathrm{a}}$ & 0.22 & 2.58 & 0.01 \\
\hline R superior longitudinal & -0.32 & 2.87 & 0.005 & -0.28 & 2.29 & 0.02 & 0.31 & 3.7 & $\mathbf{0 . 0 0 0 3 ^ { a }}$ & 0.20 & 3.24 & 0.02 \\
\hline L inferior longitudinal & -0.13 & I.1 & 0.29 & -0.08 & 0.59 & 0.56 & 0.12 & 1.41 & 0.16 & 0.22 & 2.62 & 0.01 \\
\hline
\end{tabular}

Adjustment is made for age, head motion parameters, and sex.

aSurvives Bonferroni adjustment for multiple comparisons.

Results in bold indicate those surviving Bonferroni adjustment for multiple comparisons.

sex, and for the left corticospinal and left inferior frontooccipital in unadjusted analyses only. These group differences were associated with medium to large effect sizes. The persistent ADHD also showed a nominally significant reduction in FA in the superior longitudinal fasciculi relative to the comparison group. Again, changes in radial, not axial diffusivity, accounted for these effects. A comparison of the remitted and persistent ADHD groups 

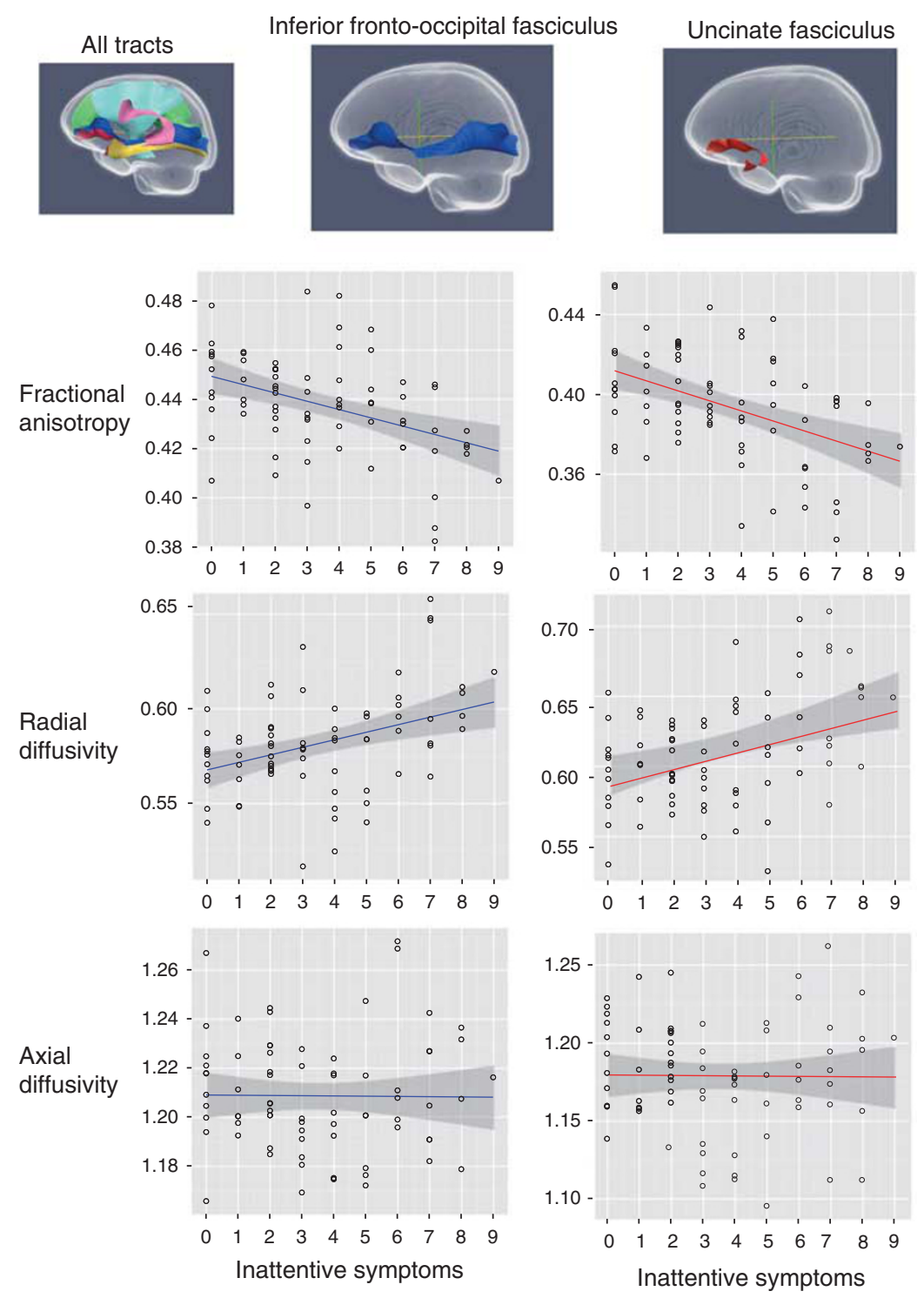

Figure I The top panel shows the II major tracts. The left fronto-occipital (in blue) and uncinate (in red) showed a significant association with inattentive symptoms that survived adjustment for multiple comparisons; the superior longitudinal fasciculus (pink); corticospinal tracts (light blue); and corpus callosum did not. Scatter plots with regression lines (and 95\% confidence intervals) show the significant association between inattention and fractional anisotropy, and radial but not axial diffusivity in these tracts.

followed the pattern of results found in the analyses based on symptom scores. Thus the persistent ADHD group had lower FA than the remitted group in adjusted analyses in the bilateral inferior fronto-occipital, the right superior longitudinal and left uncinate fasciculi (Supplementary Table 2).

The pattern of results held when the presence of comorbidity was entered as a covariate and when those who were taking psychostimulant medication regularly were excluded (Supplementary Table 3).

Finally, in tract-based analyses using childhood diagnostic status (childhood ADHD contrasted against the neveraffected comparison group) there were nominally significant reductions in FA in the bilateral inferior fronto-occipital, uncinate, and corticospinal tracts. However, none of these group difference survived adjustment for covariates and multiple comparisons (Supplementary Table 4).

\section{DISCUSSION}

We find atypical white matter microstructure among adults whose childhood symptoms of ADHD persist, delineating a major contributor to the abnormal structural connectivity seen in the disorder (Konrad and Eickhoff, 2010b). Specifically, decreased FA was found in the left inferior fronto-occipital and uncinate fasciculi that reflected primarily the severity of adult inattentive but not hyperactiveimpulsive symptoms. Whereas the persistent ADHD group differed significantly from the never-affected comparison group, the remitted ADHD group did not. The dominant change resided in diffusion perpendicular and not parallel to the axon; that is, in radial and not axial diffusivity.

The findings both replicate and extend previous studies (Casey et al, 2007; Cortese et al, 2013; Konrad et al, 2010a; 


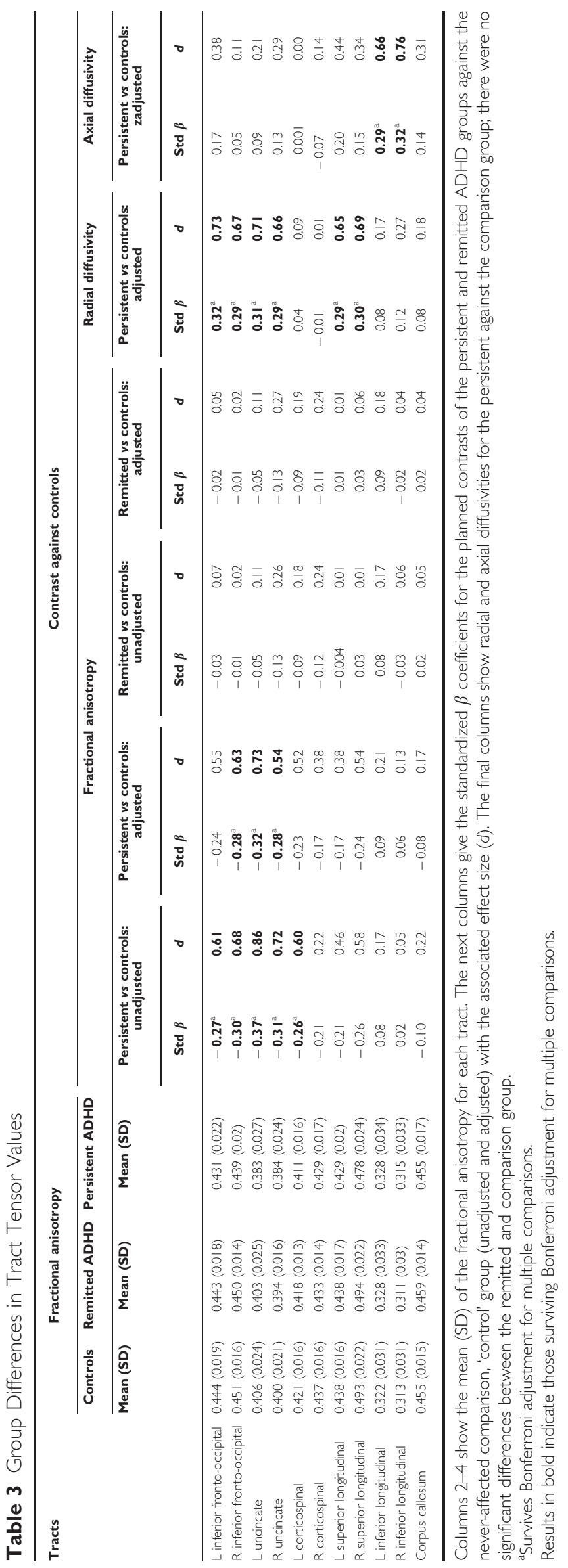

Makris et al, 2008). Reduced FA within the inferior frontooccipital tract that connects visual association cortex to prefrontal and auditory regions is a consistent finding in adult ADHD (Cortese et al, 2013; Konrad et al, 2010a). This tract is implicated in attention set-shifting abilities, semantic processing and reading, cognitive skills which are either impaired in ADHD or found in frequently comorbid learning disabilities (Doricchi et al, 2008; Epelbaum et al, 2008). Decreased FA of the uncinate fasciculus, which joins the orbitofrontal cortex and subcortical limbic regions, has also been reported both in children and adults with ADHD (Konrad et al, 2010a; Nagel et al, 2011). Given that this tract connects core components of systems generating and regulating emotions as well as the processing of reward and salience, its altered microstructure in ADHD is notable given the high prevalence of emotional dysregulation ADHD throughout the life span (Shaw et al, 2014). Fasciculi that showed decreased FA in unadjusted analyses only included the left corticospinal tract. Our study is thus consonant with prior reports of anomalies within these tracts and provides a plausible substrate for the deficits in motor control seen in ADHD, although we note that no significant association with hyperactive-impulsive symptoms was found (Cole et al, 2008; Mostofsky et al, 2006; van Ewijk et al, 2012). Indeed, the only associated with hyperactive-impulsive symptom emerged in the right superior longitudinal fasciculus. This is consonant with previous findings of decreased FA in this tract in those with persistent ADHD (Konrad et al, 2010a; Makris et al, 2008; van Ewijk et al, 2012). This tract connects the inferior parietal lobule and dorsolateral prefrontal cortex, central components of the neural systems subserving the selection of stimuli for attentional focus and working memory, and is implicated in the production of complex motor acts (Oechslin et al, 2009). In summary, the principle anomalies are identified within neural systems that support facets of attentional control (the inferior fronto-occipital fasciculus) and 'hot' cognitive-affective processes (the uncincate fasciculus), each perhaps contributing to the complex symptom profile of ADHD (Casey and Durston, 2006; Sonuga-Barke, 2005).

We found that inattention, but not hyperactivityimpulsivity, was predominately linked with white matter architectural anomalies. This is congruent with our previous finding that atypical cortical development during adolescence was detected only when considering the final endpoint of adult inattentive, but not hyperactive-impulsive symptoms (Shaw et al, 2013). Such links with adult symptom severity were not reported in the only other DTI study to include adults with childhood ADHD who had either remitted or persistent ADHD (Cortese et al, 2013). The discrepancy may reflect analytic approaches as the previous study used DSMbased categorical analyses, whereas we include analyses treating symptoms as continuous variables, thus enhancing the ability to detect symptom-related changes. Our study also benefits from advances in DTI, particularly the use of 60 noncollinear gradient directions, which allows a more precise determination of the diffusion tensor ellipsoid and thus white matter architecture than the six-direction procedure used in prior adult DTI studies.

The link between white matter microstructure and adult inattention can be interpreted in several ways. First, the disrupted structural connectivity arising from white matter 
microstructural anomalies might cause inattention. In support of this model, increases in mainly prefrontal white matter FA occur in tandem with the typical development of many cognitive skills, including working memory and information-processing abilities (Nagy et al, 2004; Tamnes et al, 2012). Moreover, the FA of long association tracts correlates with visuospatial attention in healthy adults and the severity of inattentive symptoms in those with ADHD (Tuch et al, 2005; van Ewijk et al, 2012). Alternatively, anomalies in white matter microstructure and the symptoms of ADHD might arise from a common etiological mechanism such as dysregulated modulation of cortical plasticity, in turn perhaps linked with atypical dopaminergic function (Liston et al, 2011). By this model, abnormalities of cortical plasticity that endure into adulthood, perhaps reflected by atypical neuroanatomic trajectories, could lead to both persistent symptoms and disrupted white matter microstructure as common end points. By contrast, if early childhood cortical plasticity anomalies rectify during adolescence, perhaps captured by normalizing neuroanatomic trajectories, this might result in both clinical remission and more typical adult white matter microstructure.

We can only speculate on the genomic and molecular mechanisms at play. Polymorphisms of the dopaminergic system have been associated with the persistence of childhood ADHD into adulthood, specifically of the dopamine transporter gene and dopamine D4 receptor (Franke et al, 2010). The variants implicated appear to be functional, and impact on cortical and striatal dimensions (Durston et al, 2005; Shaw et al, 2007). Dopaminergic tone may also influence white matter microarchitecture. Thus manipulations of dopamine precursors produces parallel changes in the levels of both dopamine and myelin-related proteins, and the dopamine D3 receptor is expressed in oligodendrocyte precursors (Bongarzone et al, 1998; Joseph and Dyer, 2003). In addition, genetic variation within genes controlling myelin-related protein expression have been linked with abnormal white matter structure in substance dependence, which commonly co-occurs with ADHD (Moeller et al, 2004). Thus, our findings provide another context for the interpretation of ongoing large-scale studies into the genomic architecture of ADHD.

This study can also inform future searches for trait markers for ADHD. Some have defined 'trait' markers in adult cross-sectional data through analyses based on childhood diagnoses When we employed this approach we found trends to decreased FA in multiple tracts, congruent with an earlier study (Cortese et al, 2013). However, when we use the more stringent definition of a trait marker as anomalies present among adults who have remitted, no tract level differences were found. Future studies should evaluate the possibility of static, white matter 'trait' anomalies in ADHD using prospectively acquired data.

This is the first adult DTI study in ADHD to report on the different components of FA, and finds that white matter changes were almost entirely attributable to shifts in radial, not axial, diffusivity. Given that FA is calculated from both radial and axial diffusivity, a significant difference in radial diffusivity will not always translate into a significant difference in FA, particularly when axial diffusivity does not differ significantly, as we found for some tracts. Moreover, decomposing FA into radial and axial diffusivity can cause problems both in regions of low FA and in areas where white matter tracts are likely to cross (WheelerKingshott and Cercignani, 2009). These pitfalls are mitigated in the current study by restricting analyses to regions of high FA and the spatial extent of the findings, which incorporates regions unlikely to have a high proportion of crossing fibers. Radial diffusivity represents a complex amalgam of neuronal and glial microstructure, prohibiting a simple mapping from this metric to tissue properties (Klawiter et al, 2011; Song et al, 2002; Song et al, 2005). Nonetheless, human and animal studies link changes in myelination with increases in radial, but not axial, diffusivity. As the other contributors to radial diffusivity become apparent, they may join the processes underpinning myelination as novel targets for genomic and molecular studies in ADHD.

Change in white matter detected by DTI has proved a sensitive measure of learning and the efficacy of behavioral interventions (Zatorre et al, 2012). Interventions which boost the ability to sustain attention and regulate emotions in adults have been linked with increased fractional anisotropy in the cingulum bundle, an effect driven primarily by reductions in radial diffusivity (Tang et al, 2012). Such findings suggest that changes in white matter microstructure might serve as a biomarker to help evaluate the efficacy and potentially understand the mechanisms of novel interventions.

Embedding this large cross-sectional study within a prospectively followed cohort avoids the problems inherent in retrospective recall of childhood problems. Among the limitations of the study are the higher rates of comorbidities and psychostimulant use among those with persistent ADHD, possibly contributing to the findings. Against this possibility, results held when analyses were controlled for these factors. We also excluded those who were substance dependent at the time of the study given the impact of substance dependence on white matter microstructure (Pfefferbaum and Sullivan, 2004). A final limitation is that 11 of the 75 clinical participants entered the study as adults, and their childhood diagnosis was made in the community not in the research clinic.

This study demonstrates alterations in ADHD in white matter microstructure tracking with the severity of inattention. The findings are driven by changes in radial rather than axial diffusivity. The biophysical basis of radial diffusivity is only partly understood but includes myelination, a finding that can inform future pathophysiological studies and provide potential targets for pharmacological and behavioral interventions.

\section{FUNDING AND DISCLOSURE}

The study was funded by the Intramural Program of the National Human Genome Research Institute and the National Institute of Mental Health. The authors declare no conflict of interest.

\section{REFERENCES}

Basser PJ, Mattiello J, LeBihan D (1994). Estimation of the effective self-diffusion tensor from the NMR spin echo. J Magn Reson B 103: $247-254$. 
Basser PJ, Pierpaoli C (1996). Microstructural and physiological features of tissues elucidated by quantitative-diffusion-tensor MRI. J Magn Reson B 111: 209-219.

Birnbaum HG, Kessler RC, Lowe SW, Secnik K, Greenberg PE, Leong SA et al (2005). Costs of attention deficit-hyperactivity disorder (ADHD) in the US: excess costs of persons with ADHD and their family members in 2000. Curr Med Res Opin 21: 195-205.

Bongarzone ER, Howard SG, Schonmann V, Campagnoni AT (1998). Identification of the dopamine D3 receptor in oligodendrocyte precursors: potential role in regulating differentiation and myelin formation. J Neurosci 18: 5344-5353.

Casey B, Durston S (2006). From behavior to cognition to the brain and back: what have we learned from functional imaging studies of attention deficit hyperactivity disorder? Am J Psychiatry 163: 957-960.

Casey BJ, Epstein JN, Buhle J, Liston C, Davidson MC, Tonev ST et al (2007). Frontostriatal connectivity and its role in cognitive control in parent-child dyads with ADHD. Am J Psychiatry 164: 1729-1736.

Castellanos FX, Proal E (2012). Large-scale brain systems in ADHD: Beyond the prefrontal-striatal model. Trends Cogn Sci 16: $17-26$.

Clerkin SM, Schulz KP, Berwid OG, Fan J, Newcorn JH, Tang CY et al (2013). Thalamo-cortical activation and connectivity during response preparation in adults with persistent and remitted ADHD. Am J Psychiatry 170: 1011-1019.

Coghill D, Hayward D, Rhodes S, Grimmer C, Matthews K (2014). A longitudinal examination of neuropsychological and clinical functioning in boys with attention deficit hyperactivity disorder (ADHD): improvements in executive functioning do not explain clinical improvement. Psychol Med 44: 1087-1099.

Cole W, Mostofsky S, Larson JG, Denckla M, Mahone E (2008). Age-related changes in motor subtle signs among girls and boys with ADHD. Neurology 71: 1514-1520.

Cortese S, Imperati D, Zhou J, Proal E, Klein RG, Mannuzza S et al (2013). White matter alterations at 33-year follow-up in adults with childhood attention-deficit/hyperactivity disorder. Biol Psychiatry 74: 591-598.

Doricchi F, Thiebaut de Schotten M, Tomaiuolo F, Bartolomeo P (2008). White matter (dis)connections and gray matter (dys)functions in visual neglect: Gaining insights into the brain networks of spatial awareness. Cortex 44: 983-995.

DuPaul GJ, Power JD, Anastopouls AA, Reid R (1998). ADHD Rating Scale-IV: Checklists, Norms and Clinical Interpretation. The Guilford Press: New York, NY, USA.

Durston S, Fossella J, Casey B, Hulshoff Pol H, Galvan A, Schnack H et al (2005). Differential effects of DRD4 and DAT1 genotype on fronto-striatal gray matter volumes in a sample of subjects with attention deficit hyperactivity disorder, their unaffected siblings, and controls. Mol Psychiatry 10: 678-685.

Durston S, van Belle J, de Zeeuw P (2011). Differentiating frontostriatal and fronto-cerebellar circuits in attention-deficit/ hyperactivity disorder. Biol Psychiatry 69: 1178-1184.

Epelbaum S, Pinel P, Gaillard R, Delmaire C, Perrin M, Dupont S et al (2008). Pure alexia as a disconnection syndrome: new diffusion imaging evidence for an old concept. Cortex 44: 962-974.

Faraone SV, Biederman J, Mick E (2006). The age-dependent decline of attention deficit hyperactivity disorder: a metaanalysis of follow-up studies. Psychol Med 36: 159-165.

Franke B, Vasquez AA, Johansson S, Hoogman M, Romanos J, Boreatti-Hummer A et al (2010). Multicenter analysis of the SLC6A3/DAT1 VNTR haplotype in persistent ADHD suggests differential involvement of the gene in childhood and persistent ADHD. Neuropsychopharmacology 35: 656-664.

Halperin JM, Schulz KP (2006). Revisiting the role of the prefrontal cortex in the pathophysiology of attention-deficit/hyperactivity disorder. Psychol Bull 132: 560-581.
Joseph B, Dyer CA (2003). Relationship between myelin production and dopamine synthesis in the PKU mouse brain. J Neurochem 86: 615-626.

Klawiter EC, Schmidt RE, Trinkaus K, Liang H-F, Budde MD, Naismith RT et al (2011). Radial diffusivity predicts demyelination in ex vivo multiple sclerosis spinal cords. Neuroimage 55: 1454-1460.

Konrad A, Dielentheis TF, El Masri D, Bayerl M, Fehr C, Gesierich T et al (2010a). Disturbed structural connectivity is related to inattention and impulsivity in adult attention deficit hyperactivity disorder. Eur J Neurosci 31: 912-919.

Konrad K, Eickhoff SB (2010b). Is the ADHD brain wired differently? A review on structural and functional connectivity in attention deficit hyperactivity disorder. Hum Brain Mapp 31: 904-916.

Liston C, Cohen MM, Teslovich T, Levenson D, Casey B (2011). Atypical prefrontal connectivity in attention-deficit/hyperactivity disorder: pathway to disease or pathological end point? Biol Psychiatry 69: 1168-1177.

Mackie S, Shaw P, Lenroot R, Pierson R, Greenstein DK, Nugent TF 3 rd et al (2007). Cerebellar development and clinical outcome in attention deficit hyperactivity disorder.. Am J Psychiatry 164: 647-655.

Makris N, Buka SL, Biederman J, Papadimitriou GM, Hodge SM, Valera EM et al (2008). Attention and executive systems abnormalities in adults with childhood ADHD: a DT-MRI study of connections. Cereb Cortex 18: 1210-1220.

Mattfeld AT, Gabrieli JD, Biederman J, Spencer T, Brown A, Kotte A et al (2014). Brain differences between persistent and remitted attention deficit hyperactivity disorder. Brain 137(Pt 9): 2423-2428.

Moeller FG, Hasan KM, Steinberg JL, Kramer LA, Dougherty DM, Santos RM et al (2004). Reduced anterior corpus callosum white matter integrity is related to increased impulsivity and reduced discriminability in cocaine-dependent subjects: diffusion tensor imaging. Neuropsychopharmacology 30: 610-617.

Mostofsky SH, Rimrodt SL, Schafer JG, Boyce A, Goldberg MC, Pekar JJ et al (2006). Atypical motor and sensory cortex activation in attention-deficit/hyperactivity disorder: a functional magnetic resonance imaging study of simple sequential finger tapping. Biol Psychiatry 59: 48-56.

Nagel BJ, Bathula D, Herting M, Schmitt C, Kroenke CD, Fair D et al (2011). Altered white matter microstructure in children with attention-deficit/hyperactivity disorder. J Am Acad Child Adolesc Psychiatry 50: 283-292.

Nagy Z, Westerberg H, Klingberg T (2004). Maturation of white matter is associated with the development of cognitive functions during childhood. J Cogn Neurosci 16: 1227-1233.

Nigg JT, Casey BJ (2005). An integrative theory of attention-deficit/ hyperactivity disorder based on the cognitive and affective neurosciences. Dev Psychopathol 17: 785-806.

Oechslin MS, Imfeld A, Loenneker T, Meyer M, Jäncke L (2009). The plasticity of the superior longitudinal fasciculus as a function of musical expertise: a diffusion tensor imaging study. Front Hum Neurosci 3: 76.

Pfefferbaum A, Sullivan EV (2004). Disruption of brain white matter microstructure by excessive intracellular and extracellular fluid in alcoholism: evidence from diffusion tensor imaging. Neuropsychopharmacology 30: 423-432.

Pierpaoli C, Walker L, Irfanoglu M, Barnett A, Basser P, Chang L et al (2010). TORTOISE: an integrated software package for processing of diffusion MRI data. ISMRM 18th Annual Meeting, Stockholm, Sweden, \#1597.

Pingault JB (2011). Childhood trajectories of inattention and hyperactivity and prediction of educational attainment in early adulthood: a 16-year longitudinal population-based study. Am J Psychiatry 168: 1164. 
Plessen KJ, Bansal R, Zhu H, Whiteman R, Amat J, Quackenbush GA et al (2006). Hippocampus and amygdala morphology in attention-deficit/hyperactivity disorder. Arch Gen Psychiatry 63: 795-807.

Proal E, Reiss PT, Klein RG, Mannuzza S, Gotimer K, RamosOlazagasti MA et al (2011). Brain gray matter deficits at 33-year follow-up in adults with attention-deficit/hyperactivity disorder established in childhood. Arch Gen Psychiatry 68: 1122-1134.

Rajendran K, Rindskopf D, O’Neill S, Marks DJ, Nomura Y, Halperin JM (2013). Neuropsychological functioning and severity of ADHD in early childhood: A four-year cross-lagged study. J Abnorm Psychol 122: 1179.

Reich W (2000). Diagnostic interview for children and adolescents (DICA). J Am Acad Child Adolesc Psychiatry 39: 59-66.

Shaw P, Gornick M, Lerch J, Addington A, Seal J, Greenstein D et al (2007). Polymorphisms of the dopamine d4 receptor, clinical outcome, and cortical structure in attention-deficit/hyperactivity disorder. Arch Gen Psychiatry 64: 921-931.

Shaw P, Malek M, Watson B, Greenstein D, de Rossi P, Sharp W (2013). Trajectories of cerebral cortical development in childhood and adolescence and adult attention-deficit/hyperactivity disorder. Biol Psychiatry 74: 599-606.

Shaw P, Stringaris A, Nigg J, Leibenluft E (2014). Emotion dysregulation in attention deficit hyperactivity disorder. Am J Psychiatry 171: 276-293.

Smith SM, Jenkinson M, Johansen-Berg H, Rueckert D, Nichols TE, Mackay CE et al (2006). Tract-based spatial statistics: voxelwise analysis of multi-subject diffusion data. Neuroimage 31: 1487-1505.

Song S-K, Sun S-W, Ramsbottom MJ, Chang C, Russell J, Cross AH (2002). Dysmyelination revealed through MRI as increased radial (but unchanged axial) diffusion of water. Neuroimage 17: 1429-1436.

Song S-K, Yoshino J, Le TQ, Lin S-J, Sun S-W, Cross AH et al (2005). Demyelination increases radial diffusivity in corpus callosum of mouse brain. Neuroimage 26: 132-140.

Sonuga-Barke EJ (2005). Causal models of attention-deficit/ hyperactivity disorder: from common simple deficits to multiple developmental pathways. Biol Psychiatry 57: 1231-1238.
Tamnes CK, Fjell AM, Westlye LT, Østby Y, Walhovd KB (2012). Becoming consistent: developmental reductions in intraindividual variability in reaction time are related to white matter integrity. J Neurosci 32: 972-982.

Tang Y-Y, Lu Q, Fan M, Yang Y, Posner MI (2012). Mechanisms of white matter changes induced by meditation. Proc Natl Acad Sci USA 109: 10570-10574.

Tuch DS, Salat DH, Wisco JJ, Zaleta AK, Hevelone ND, Rosas HD (2005). Choice reaction time performance correlates with diffusion anisotropy in white matter pathways supporting visuospatial attention. Proc Natl Acad Sci USA 102: 12212-12217. van Ewijk H, Heslenfeld DJ, Zwiers MP, Buitelaar JK, Oosterlaan J (2012). Diffusion tensor imaging in attention deficit/hyperactivity disorder: A systematic review and meta-analysis. Neurosci Biobehav Rev 36: 1093-1106.

van Lieshout M, Luman M, Buitelaar J, Rommelse N, Oosterlaan J (2013). Does neurocognitive functioning predict future or persistence of ADHD? A systematic review. Clin Psychol Rev 33: 539-560.

Wang Y, Gupta A, Liu Z, Zhang H, Escolar ML, Gilmore JH et al (2011). DTI registration in atlas based fiber analysis of infantile Krabbe disease. Neuroimage 55: 1577-1586.

Wheeler-Kingshott CA, Cercignani M (2009). About 'axial' and 'radial' diffusivities. Magn Reson Med 61: 1255-1260.

Zatorre RJ, Fields RD, Johansen-Berg H (2012). Plasticity in gray and white: neuroimaging changes in brain structure during learning. Nat Neurosci 15: 528-536.

Zhang H, Avants BB, Yushkevich PA, Woo JH, Sumei W, McCluskey LF et al (2007). High-dimensional spatial normalization of diffusion tensor images improves the detection of white matter differences: an example study using amyotrophic lateral sclerosis. IEEE Trans Med Imaging 26: 1585-1597.

Zhang H, Awate SP, Das SR, Woo JH, Melhem ER, Gee JC et al (2010). A tract-specific framework for white matter morphometry combining macroscopic and microscopic tract features. Med Image Anal 14: 666-673.

Zhang H, Yushkevich P, Rueckert D, Gee J (2009). A computational DTI template for aging studies. Proc Intl Soc Mag Reson Med 217: 3230 .

Supplementary Information accompanies the paper on the Neuropsychopharmacology website (http://www.nature.com/npp) 